\title{
RULED SURFACES AND THE ALBANESE MAPPING
}

\author{
BY ARTHUR MATTUCK ${ }^{1}$
}

Communicated March 17, 1969

1. Much of the classical theory of algebraic curves is summarized by saying there is a map $C(n) \rightarrow J$ from the $n$-fold symmetric product of the curve $C$ into an abelian variety $J$, the Jacobian, and the fibers are projective spaces (representing the linear systems of degree $n$ ). For algebraic surfaces there is an analogous map $V(n) \rightarrow A$ from the $n$-fold symmetric product of the surface $V$ to its Albanese variety. The fibers are irreducible and regular if $n$ is large, but it has been a long open question whether they are rational, or ever can be.

THEOREM. Let $V$ be a complete nonsingular surface in characteristic zero, and let $q$ denote the dimension of its Albanese variety $A$. If for some $n>q$ the general fiber of the morphism $V(n) \rightarrow A$ is a rational variety, then $V$ is a ruled surface.

By the "general" fiber we mean as usual that there is an open set in $A$ over which all fibers have the indicated property. If $V$ is ruled, i.e., birationally equivalent to the product $P^{1} \times C$ of a projective line and a curve $C$, then the general fiber is rational for all $n$ : for this converse to the theorem, one needs only the quoted result for curves plus the remark that then the Albanese variety of $V$ is just the Jacobian of $C$. A proof of the theorem when $q=0$ was the subject of an earlier paper [2], some of whose ideas recur here. There is also overlap with a recent (independent) proof by Mumford [3] that the rational equivalence ring is not of finite type; both proofs use the idea of bounding the dimension of the zero-locus of a 2 -form.

\section{A generic smoothness lemma. We need the}

LEMMA. Let $f: X \rightarrow Y$ be a dominating morphism of varieties in characteristic zero, with $X$ nonsingular and projective. Then $f$ has maximal rank along the general fiber $F_{y}$, so $F_{y}$ is nonsingular.

PRoof. The lemma is local on $Y$; by Noether normalization we may reduce to the case where $Y$ is affine $r$-space, with coordinate functions $x_{1}, \cdots, x_{r}$. As $a_{1}$ varies over the (algebraically closed) ground field, the zeros of $x_{1}-a_{1}$ on $X$ give a linear system of divisors on $X$; by Bertini's theorem, a general member-say $X_{1}$-is a disjoint union of

1 Research supported in part by the National Science Foundation. 
nonsingular varieties; each has multiplicity one and no embedded components. In the same way the zeros of $x_{2}-a_{2}$ on $X_{1}$ give a linear system on $X_{1}$ whose general member is nonsingular, etc. Continuing, we see that there exist fibers $f^{-1}(a)$ along which $f$ is of maximal rank. This then follows for the general fiber since $f$ being of maximal rank is an open condition on $Y$ : it is clearly open on $X$ and $f$ is proper.

For the proof of our theorem, we may replace the singular variety $V(n)$ by a canonical desingularization, the Hilbert scheme $H(n)$. This is [1] a nonsingular $2 n$-dimensional projective variety with a birational morphism $h_{n}: H(n) \rightarrow V(n)$. This gives a corresponding morphism $H(n) \rightarrow A$, whose general fiber is pure $(2 n-q)$-dimensional by dimension theory, and it is easily seen that $h_{n}$ is an isomorphism on an open subset of each component of the general fiber. Thus our hypotheses together with the lemma imply that

(1) For some $n>q$, the general fiber of $H(n) \rightarrow A$ is a nonsingular rational variety.

3. Differential forms of weight $r$. Let $X$ be an $n$-dimensional variety, with function field $K=k(X)$, and let $E^{p}$ be the $K$-space of $p$-forms on $X$; then the $\left(m_{1}, \cdots, m_{r}\right)$-forms are the elements of $E^{m_{1}} \otimes \cdots \otimes E^{m_{r}} ;$ if all $m_{i}=m$, they are called the $m$-forms of weight $r$. These forms are holomorphic at $p \in X$ if the coefficients are holomorphic when the form is written in terms of $d x_{1}, \cdots, d x_{n}$, where the $x_{i}$ are local parameters at $p$. Thus if $X$ is also complete, the number of independent global holomorphic $\left(m_{1}, \cdots, m_{r}\right)$-forms is given by $h^{m_{1}}, \ldots, m_{r}=\operatorname{dim} H^{0}\left(X, \Omega^{m_{1}} \otimes \cdots \otimes \Omega^{m_{r}}\right)$, where $\Omega^{k}$ is the sheaf of holomorphic $k$-forms. If $m_{i}=n$ for all $i, h^{n}, \ldots, n$ is traditionally written $P_{r}(X)$, and called the rth plurigenus of $X$. These are all birational invariants for $X$ complete nonsingular, for

(2) If $f: X \rightarrow Y$ is a dominating, separable, rational map of complete nonsingular varieties, then $h^{m_{1}}, \ldots, m_{r}(X) \geqq h^{m_{1}}, \ldots . m_{r}(Y)$.

The reasoning is classical. If $\alpha$ is a holomorphic form on $Y$, then $f^{*} \alpha$ is a form of the same type on $X$ which is nonzero (separability); holomorphic outside a locus of codimension $\geqq 2$ (the fundamental locus), therefore holomorphic everywhere (nonsingularity of $X$ ).

Proposition. If $X$ is a nonsingular rational (or unirational) variety, then $h^{m_{1}} \ldots \ldots m_{r}(X)=0$ for all $\left(m_{1}, \cdots, m_{r}\right) \neq(0, \cdots, 0)$.

Proof. It suffices to prove this when $X$ is projective $n$-space, by (2). Let $x_{0}, \cdots, x_{n}$ be projective coordinates, and $g: A-(0) \rightarrow X$ the usual map of affine $(n+1)$-space minus the origin onto projective 
space. If $\alpha$ is a holomorphic form on $X$, then $g^{*} \alpha$ is holomorphic on $A-(0)$, therefore on $A$ since $\operatorname{cod}(0) \geqq 2$. Written in terms of $x_{i}$, its coefficients are thus polynomials; since it is invariant under the automorphisms of $A$ defined by $x_{i} \rightarrow c x_{i}$, we get all $m_{i}=0$.

4. Proof of the theorem. By a well-known result (see e.g. [4]), if $V$ is a complete nonsingular surface in characteristic zero, then $V$ is ruled if and only if $P_{r}(V)=0$ for all $r>0$. So we prove:

(3) If for some $r, V$ carries a nonzero holomorphic 2-form $\phi$ of weight $r$, then the general fiber of $H(n) \rightarrow A$ is not rational.

Let $V[n]$ be the $n$-fold product; given such a $\phi$, then

$$
\Phi=\phi_{1}+\cdots+\phi_{n}, \quad \phi_{i}=\operatorname{pr}_{i}^{*} \phi
$$

is a holomorphic 2 -form of weight $r$ on $V[n]$; since it is invariant under the symmetric group $S_{n}$, it is the lifting of a form on $V(n)$, and this in turn may be carried over to $H(n)$. We use the same letter $\Phi$ for any of these forms. If we grant that $\Phi$ is holomorphic on $H(n)$ this will be proved later-then the restriction $\Phi_{F}$ of $\Phi$ to a (nonsingular) general fiber $F$ of $H(n) \rightarrow A$ gives a holomorphic 2-form of weight $r$ on $F$. If $F$ were rational, then $\Phi_{F}=0$ by the proposition. But if we pull things back to $V[n]$, this contradicts

(5) If $n>q$, the restriction of $\Phi$ to the general fiber of $V[n] \rightarrow A$ is not zero.

Proof of (5). Let $p=\left(p_{1}, \cdots, p_{n}\right)$ be a general point of $V[n]$, $F$ the fiber through it, $T_{p, F}$ the tangent space to $F$ at $p$. We say

(6) $\sigma_{i}: T_{p, F} \rightarrow T_{p_{i}, V}$ is onto for all $i\left(\sigma_{i}=d\left(p r_{i} \mid F\right)\right)$.

Namely, let $S_{i}$ be the closure of the set of $q \in V[n]$ which are either singular points of the fiber $F_{q}$ through them or else where $\sigma_{i}$ is not onto, i.e., has rank $\leqq 1$. Since $\operatorname{dim} T_{p, F}=2 n-q>n$, this space cannot be mapped to a 1 -dimensional space by each of the $n$ maps $\sigma_{i}$. Say $\sigma_{1}$ has rank 2 ; introducing coordinates, we see that rank $\sigma_{1}=2$ in a neighborhood of $p$. Thus $p \notin S_{1}$, so $S_{1}$ is a proper closed set. It follows by symmetry that $S_{i}$ is a proper closed set, and therefore $p \notin S_{i}$ for any $i$, which is the assertion (6).

From (6) it follows that for each $i$, we can choose vectors $t_{i}, t_{i}^{\prime}$ in $T_{p, F}$ whose images under $\sigma_{i}$ are independent. Taking general linear combinations of the $t_{i}$ and of the $t_{i}^{\prime}$, we conclude

(7) There are vectors $t, t^{\prime}$ in $T_{p, r}$ such that $\sigma_{i}(t)$ and $\sigma_{i}\left(t^{\prime}\right)$ are independent for all $i$. 
We now prove (5). Choose $x$ and $y$ to be local parameters at each point $p_{i}$; thus $\phi=\left.g(d x d y)\right|^{\otimes_{r}}$, where $g\left(p_{i}\right)=a_{i} \neq 0$ since $p_{i}$ is a general point of $V$. By $(7),\left\langle d x d y,\left(\sigma_{i}(t), \sigma_{i}\left(t^{\prime}\right)\right)\right\rangle=b_{i} \neq 0$. On the space $T_{p, V[n]}$, by (4) the form $\Phi=\left.\sum a_{i}\left(d x_{i} d y_{i}\right)\right|^{\otimes r}$. If $\Phi$ were 0 when restricted to the subspace $T_{p, F}$, then for $e, e^{\prime} \in T_{p, F}$,

$$
\left\langle\Phi,\left(e, e^{\prime}, t, t^{\prime}, \cdots, t, t^{\prime}\right)\right\rangle=\sum a_{i}\left\langle d x_{i} d y_{i},\left(e, e^{\prime}\right)\right\rangle b_{i}^{r-1}=0 .
$$

Our hypothesis is that $\operatorname{dim} T_{p, F}>n$. If we put in $n+1$ linearly independent vectors for $e^{\prime}$, we get from the above $n+1$ independent linear equations in $2 n$ variables (the coefficients of $e$ ), having at least $n+1$ independent solution vectors $e$, a contradiction.

We still must show $\Phi$ is holomorphic on $H(n)$. Let $X$ be the normalization of $H(n)$ in the function field of $V[n]$. Then the symmetric group $S_{n}$ acts as automorphisms of $X$ and $H(n)$ is the quotient $X / S_{n}$. Since $\Phi$ is holomorphic on $V[n]$, when viewed as a differential $\Phi^{\prime}$ on the normal and birationally equivalent variety $X$, it will have no poles. Therefore on $H(n)$, its trace $\operatorname{tr}_{X \mid H(n)} \Phi^{\prime}$ will also have no poles; but $(1 / n !) \operatorname{tr} \Phi^{\prime}=\Phi$.

\section{REFERENCES}

1. J. Fogarty, Algebraic families on an algebraic surface, Amer. J. Math. 90 (1968), 511-521.

2. A. Mattuck, On the symmetric product of a rational surface, Proc. Amer. Math. Soc. 21 (1969), 638-688.

3. D. Mumford, Rational equivalence of 0-cycles on surfaces, Kyoto J. Math. (to appear).

4. I. R. Safarevič et al., Algebraic surfaces, Trudy Mat. Inst. Steklov. $75(1965)=$ Proc. Steklov. Inst. Math. 75 (1965).

Massachusetts Institute of Technology, Cambridge, Massachusetts 02139 